\title{
A WATERMARKING SYSTEM FOR TEACHING STUDENTS TO RESPECT INTELLECTUAL PROPERTY RIGHTS
}

\author{
Maria Chroni, Angelos Fylakis, and Stavros D. Nikolopoulos \\ Department of Computer Science, University of Ioannina, GR-45110 Ioannina, Greece \\ \{mchroni, afylakis, stavros\}@cs.uoi.gr
}

\begin{abstract}
Keywords: Watermarking Techniques; Intellectual Property Rights; Teaching IP; Educational Tools; Visual Representations; Learning Process; Color Images; 2D-representations of Permutations; Algorithms.

Abstract: $\quad$ Our work proposes a watermarking system for supporting the teaching process for educating students to respect intellectual property rights. In particular, we propose an educational tool, which we named WaterIP, that can be efficiently used by students to enable them to perceive how to protect their own ideas and how to respect others' intellectual property. Our system uses an efficient technique for watermarking images by exploiting certain properties of a specific 2D representation of permutations, it has a friendly graphical user interface and shows interesting performance figures. The system provides students with two main working levels: (I) the student creates a secret key, i.e., the watermark, and embeds it into his own image, and (II) he makes the marks of a watermarked image visible in order to later extract the watermark from it using only, for pedagogical reasons, a ruler and a pencil. The watermarking method behind WaterIP can be applied to all educational levels beginning from early childhood up to preliminary and high schools. We demonstrate the educational effectiveness of our WaterIP system by presenting ways of how it can be applied in class and show that WaterIP helps to understand what intellectual property rights really stand for.
\end{abstract}

\section{INTRODUCTION}

As internet technology becomes an indispensable tool for everyday life, it is more important than ever for educational reform which favours the establishment of a culture where the notion of intellectual property is respected by the people.

In a synchronous context of education, teachers need to have pedagogical and technological content knowledge of intellectual property if they are to incorporate it into their learning programmes to teach students to respect others' intellectual property and protect their own ideas. In such a context, the pedagogical tools are constantly changing as the world in which teaching is situated evolves. Hence, pedagogical tools that support not only the teaching but also ideas about intellectual property rights are developing within the technological world.

A technological tool that supports and helps both teachers and students to understand, protect and respect intellectual property is of great importance, useful, and valuable. The educational value of such a tool is mainly based on the technology used, and also on the technique or method adopted for the design and implementation of the tool.
Watermarking is a technique that is currently being studied to prevent or discourage piracy and deter unauthorized copying of digital media. It incorporates many important technological and theoretical properties which enable us to design an efficient educational tool, with pedagogic value, for teaching intellectual property rights inside the classroom.

We next briefly describe the main idea behind the watermarking technique, some issues about intellectual property rights (IP), the motivation of our work, and our contribution which is an educational watermarking tool for teaching IP.

Watermarking. Digital watermarking (or, simply, watermarking) is a technique for protecting the intellectual property of a digital object; the idea is simple: a unique identifier, which is called watermark, is embedded into a digital object which may be used to verify its authenticity or the identity of its owners (Grover, 1997; Collberg and Nagra, 2010). A digital object may be audio, picture, video, or software, and the watermark is embedded into object's data through the introduction of errors not detectable by human perception (Cox et al., 1996); note that, if the object is copied then the watermark also is carried in the copy. 
The watermarking problem can be described as the problem of embedding a watermark $w$ into an object $I$ and, thus, producing a new object $I_{w}$, such that $w$ can be reliably located and extracted from $I_{w}$ even after $I_{w}$ has been subjected to transformations (Collberg and Nagra, 2010); for example, compression in case the object is an image.

There are two general types of watermarking, namely, visible and invisible watermarking. In visible watermarking, information (i.e., the watermark) is visible in the object, i.e., audio, image, or video. For example, when a television broadcaster adds its logo to the corner of transmitted video, this is a visible watermark. Moreover, there are many watermark tools that allow us to quickly and easily protect our images with a visible watermark; with the many watermarking options available, we are able to personalize our images in a variety of ways. In invisible watermarking, information is added as digital data to object, but it cannot be perceived as such (although it may be possible to detect that some amount of information is hidden in the object).

It is worth noting that although digital watermarking has made considerable progress and become a popular technique for copyright protection of multimedia information (Cox et al., 1996; Tamada et al., 2004), research on watermarking tool designing for educational purposes has not yet received sufficient attention.

Intellectual Property. The term intellectual property (IP) refers to a creation of a mind for which a set of exclusive rights are recognized (Raysman et al., 1999). That creation may have any form possible; for example, it may be a work of art, an invention, literary or artistic work, a discovery or even a phrase. More precisely, IP can be divided into two categories: industrial property, which includes inventions (patents), trademarks, industrial designs, and geographic indications of source; and copyright, which includes literary and artistic works such as novels, poems, plays, films, musical works, drawings, paintings, photographs, sculptures, and architectural designs.

The objective of recognizing intellectual property is to encourage innovation. That is because people won't have the incentive to create if they are not legally protected in order to get the social value that they deserve from their creations (Lemley, 2005). Of course the world's evolution and economic growth depends on creations and inventions and that makes intellectual property such an important and vital aspect (Jain et al., 2009).

There used to be laws protecting intellectual material, but it was not until the 19th century that the term "Intellectual Property" was used for the first time.
The first modern usage of the term, goes back to 1867 when the North German Confederation granted legislative power over the protection of intellectual property. In 1893, the United International Bureaux for the Protection of Intellectual Property was established in Berne. Later, in 1960 this organization was relocated to Geneva, until 1967 when it was succeeded with the establishment of the World Intellectual Property Organization as an agency of the United Nations. That was also the time when the term became also popular in the United States (Lemley, 2005).

Over the last years the internet has been expanding very rapidly and, thus, information is now spread freely, easily and cost-efficiently and that gives a greater importance to intellectual property. Because of the internet, the distribution of intellectual material went out of control. Just the fact that nearly every intellectual material that is produced today is published in digital form or can be transformed into digital form means that it can be easily transmitted free via the internet, without any permission from the creator.

All that urged the adoption of new laws and the development of systems for the protection of intellectual property (Davis, 1997). But still the cyberspace is chaotic nowadays and that makes it extremely difficult to have any kind of control over it. The figures talk by themselves; according to IFPI (International Federation of the Phonographic Industry) 95\% of music downloads are pirated. What is more, a survey from Digital Life America showed as that things aren't any better for the movies. If we also take into account the fact that the internet population is consisted of nearly seven billion we may realize that its power is greater than the law and the systems for protection. And that's where education comes to place.

Motivation. We strongly believe that the best way to gain people's respect towards intellectual property rights is to start from the roots. Respecting intellectual property should be within a person's morals, and something like that can be acquired by a person during his early education. We also believe that students should not only be taught theoretically what intellectual property is, but they should also have an experience in order to be better motivated to learn about this aspect; that is what our work suggests: motivating students through the act of claiming a property using a watermarking technique provided by a friendly and easy to understand manner through our watermarking system. After such an experience a student will realize that intellectual property is a matter that concerns him as well. Thus, he will pay more attention at his teacher talking about it and actually make the respect towards intellectual property part of his character. 
Contribution. Based on the above motivation, we propose a watermarking system supporting the teaching process for educating students to respect intellectual property rights. In particular, we propose an educational tool, which we named WaterIP, that can be efficiently used by students to enable them to consider how to protect their own ideas. Moreover, WaterIP can be incorporated into school learning programmes to teach students to respect others' intellectual property rights.

Our system uses an efficient technique for watermarking images and provides students with two main working levels corresponding to two main components:

(I) The first component allows a student to create a secret key (i.e., the watermark) and select a picture $I$ in which he wants to embed the watermark; in our system the watermark $w$ consists of 6 distinct numbers from 1 to 6 , and is embedded into the original picture $I$ resulting the watermarked picture $I_{w}$.

(II) The second component is responsible for making the marks of a watermarked image $I_{w}$ visible to the student so that he will be able to easily extract the watermark $w$ by hand; in particular, the system returns the marked picture $I_{m}$ to the student and he extracts the watermark from $I_{m}$ using only, for pedagogical reasons, a ruler and a pencil.

We would like to point out that our system could have had a third component for extracting the watermark from the watermarked image but we chose not to include it because we consider important, for pedagogical reasons, that the student must participate interactively in the process of proving ownership.

The usability of the system is based on a watermarking technique used through a friendly graphical user interface. Using it the student can easily produce his watermark $w$ using his mouse without making any mistakes. He can also choose an image $I$ from his computer and he can either embed a watermark into $I$ resulting the watermarked image $I_{w}$ or make the marks of $I_{w}$ visible so that he will be able to prove to his teacher that the picture belongs to him.

The method behind the WaterIP tool can be applied to all educational levels, as part of different lesson plans, beginning from early childhood; teachers of preschools can demonstrate the system to preschool learners by uploading an image or photo and set them ethical dilemmas concerning the proper use of that image or photo, while teachers of preliminary and high schools can go on more complex meanings and explain learners the concepts of watermarking, embedding, extracting, permutation, image analysis, etc.
In order to demostrate the operational efficiency of our WaterIP system, we first present a scenario of two classmates in which both claim the ownership of the same image and show how our system helps both to understand what the intellectual property rights really stand for, and then we recommend a lesson plan of how our WaterIP system can be efficiently applied in groups inside a classroom during a course.

Our system has optimal time and space performance. Let $N \times M$ be the size of the input image, that is, the number of pixels of both the original image $I$ and the watermarked image $I_{w}$. The total asymptotic time performance of our system is of order $n \log n$ for decompressing the input image $I$ from JPEG or JIF format to raw raster format, of order $N+M$ for embedding the watermark $w$ into $I$, and of order $N \times M$ for marking the image $I_{w}$ and producing the image $I_{m}$, where $n=N \times M$. Moreover, the extra space needed by the system is constant since it uses only some extra auxiliary variables.

Road Map. The paper is organized as follows. In Section 2 we present efficient representations of the two main objects of our watermarking system: the permutations and the digital color images. In Section 3 we describe the main functions of the proposed watermarking system. In Section 4 we show a typical use of our system inside the classroom, while in Section 5 we conclude the paper and discuss possible future extensions.

\section{THEORETICAL TOOLS}

In this section we present representations of the two main objects of our watermarking system: the permutations and the digital color images. In particular, we propose a $2 \mathrm{D}$ representation of permutations and give a $3 \mathrm{D}$ representation of color images.

\subsection{Permutations}

Informally, a permutation of a set of objects $S$ is an arrangement of those objects into a particular order, while in a formal (mathematical) way a permutation of a set of objects $S$ is defined as a bijection from $S$ to itself (i.e., a map $S \rightarrow S$ for which every element of $S$ occurs exactly once as image value).

Permutations may be represented in many ways. The most straightforward is simply a rearrangement of the elements of the set $S$, as in the example of Figure 1 , where $S$ is the set $N_{6}=\{1,2, \ldots, 6\}$; in this way we think of the permutation $\pi=(2,5,3,1,6,4)$ as a rearrangement of the elements of the set $N_{6}$ such 
that "1 goes to 2", "2 goes to 5", "3 goes to 3", "4 goes to 1", and so on (Sedgewick and Flajolet, 1996; Golumbic, 1980).

$$
\begin{array}{rrrrrrr}
\text { index } & 1 & 2 & 3 & 4 & 5 & 6 \\
\hline \text { permutation } & 2 & 5 & 3 & 1 & 6 & 4
\end{array}
$$

Figure 1: A straightforward representation of the permutation $\pi=(2,5,3,1,6,4)$.

Based on the above representation, we can also think of a permutation $\pi=\left(\pi_{1}, \pi_{2}, \ldots, \pi_{n}\right)$ over the set $N_{n}=\{1,2, \ldots, n\}$ as a sequence $\left(\pi_{1}, \pi_{2}, \ldots, \pi_{n}\right)$ of the elements of the set $N_{n}$; so, for example, the permutation of Figure 1 has $\pi_{1}=2, \pi_{2}=5, \ldots, \pi_{6}=$ 4 , and $\pi_{1}^{-1}=4, \pi_{2}^{-1}=1, \ldots, \pi_{6}^{-1}=5$ (Golumbic, 1980).

2D Representation of Permutations. Given a permutation $\pi$ over the set $N_{n}=\{1,2, \ldots, n\}$, we first define a two-dimensional representation (2Drepresentation) of the permutation $\pi$ that is useful for studying properties which help us to define, later, a more suitable representation of $\pi$ for efficient use in our watermarking system.

In this representation, the elements of the permutation $\pi=\left(\pi_{1}, \pi_{2}, \ldots, \pi_{n}\right)$ are mapped in specific cells of an $n \times n$ matrix $A$ as follows:

$$
\begin{aligned}
& \text { - integer } i \longrightarrow \text { entry } A\left(\pi_{i}^{-1}, i\right) \\
& \text { or, equivalently, }
\end{aligned}
$$

- the cell at row $i$ and column $\pi_{i}$ is labeled by the number $\pi_{i}$, for each $i=1,2, \ldots, n$.

Figure 2 shows the 2D representation of the permutation $\pi=(2,5,3,1,6,4)$.

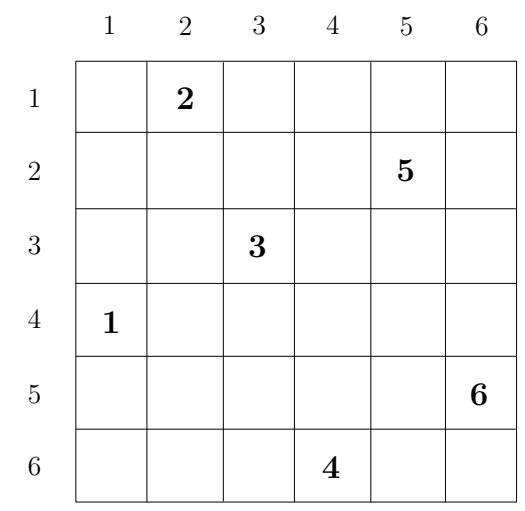

Figure 2: A 2D representation of $\pi=(2,5,3,1,6,4)$.

Note that, there is one label in each row and in each column, so each cell in the matrix $A$ corresponds to a unique pair of labels; see, (Sedgewick and Flajolet,
1996) for a long bibliography on permutation representations and also in (author's paper) for a DAG representation.

2DM Representation of Permutations. Based on the previous $2 \mathrm{D}$ representation of a permutation, we next propose a two-dimensional marked representation (2DM representation) of a permutation which is an efficient tool for watermarking images and also incorporate pedagogical and teaching issues (or, properties).

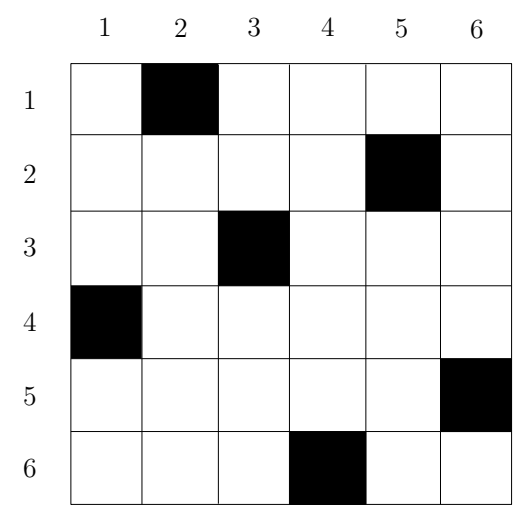

Figure 3: A 2DM representation of $\pi=(2,5,3,1,6,4)$.

In our 2DM representation, a permutation $\pi$ over the set $N_{n}=\{1,2, \ldots, n\}$ is represented by an $n \times n$ matrix $A$ as follows:

- the cell at row $i$ and column $\pi_{i}$ is marked by a specific symbol, for each $i=1,2, \ldots, n$.

Figure 3 shows the 2D marked representation of the permutation $\pi$. Note that, as in the $2 \mathrm{D}$ representation, there is also one symbol in each row and in each column of the matrix $A$.

We next present an algorithm which extracts the permutation $\pi$ from its $2 \mathrm{DM}$ representation matrix. More precisely, let $\pi$ be a permutation over $N_{n}$ and let $A$ be the 2DM representation matrix of $\pi$ (see, Figure 3 ); given the matrix $A$, we can easily extract $\pi$ from $A$ in linear time (in the size of matrix $A$ ) by the following algorithm:

Algorithm Extract_ $\pi \_$from_2DM

Input: the 2DM representation matrix $A$ of $\pi$;

Output: the permutation $\pi$;

1. For each column $i$ of matrix $A, 1 \leq i \leq n$, do: find the marked cell $j$ in the column $i$, and set $i$ in cell $A(j, i)$ and 0 in all other cells;

2. For each row $i$ of matrix $A, 1 \leq i \leq n$, do: find the cell $j$ in the row $i$ with value not equal to 0 , and set $\pi_{i} \leftarrow A(i, j)$;

3. Return the permutation $\pi$; 
It is easy to see that, the resulting matrix $A$ after the execution of Step 1 is the $2 \mathrm{D}$ representation matrix of the permutation $\pi$. Reading the positive numbers from top row to bottom row of $A$ gives back the permutation $\pi$ (Step 2).

\subsection{Color Images}

A digital image is a numeric representation of a 2dimensional image; it has a finite set of values, called picture elements or pixels, that represent the brightness of a given color at any specific point in the image (Gonzalez and Woods, 2007).

A digital image contains a fixed number of rows and columns of pixels which are usually stored in computer memory as a two-dimensional matrix $I$ of numeric values; in our system the numeric values are integers from 0 to 255 . When we say that an image has a resolution of $N \times M$ we mean that its two-dimensional matrix $I$ contains $N$ rows and $M$ columns and the value of each entry $I(i, j)$, i.e., the value of each pixel, is an integer $k_{0}$ (grayscale image), or a triple of integers $\left(k_{1}, k_{2}, k_{3}\right)$ (color image), $0 \leq k_{0}, k_{1}, k_{2}, k_{3} \leq 255$.

There are several models used for representing color. In our system, we use the $R G B$ model; it is an additive color model in which red, green, and blue light is added together in various ways to reproduce a broad array of colors. The name of the model comes from the initials of the three additive primary colors, Red, Green, and Blue (Gonzalez and Woods, 2007; Pascale, 2003).

The range of colors can be represented on the Cartesian 3-dimensional system as a cube with the following characteristics:

- on the $x$-axis ( $R$-axis) we have the brightness of the red colour,

- on the $y$-axis ( $G$-axis) we have the brightness of the green colour, and

- on the $z$-axis ( $B$-axis) we have the brightness of the blue colour.

Figure 4 shows the 3D topology of the colors. For example, the white color $(255,255,255)$ is located in the front upper right point of the color cube.

In our system, since a color is a triple of integers $(x, y, z)$, a digital image $I$ of resolution $N \times M$ (i.e., it contains $N$ rows and $M$ columns of pixels) is stored in a three-dimensional matrix $\operatorname{Img}$ of size $N \times M \times 3$ as follows:

- if the pixel $I(i, j)$ of the image $I$ has $(x, y, z)$ color, then $\operatorname{Img}(i, j, 1)=x, \operatorname{Img}(i, j, 2)=y$, and $\operatorname{Img}(i, j, 3)=z$.

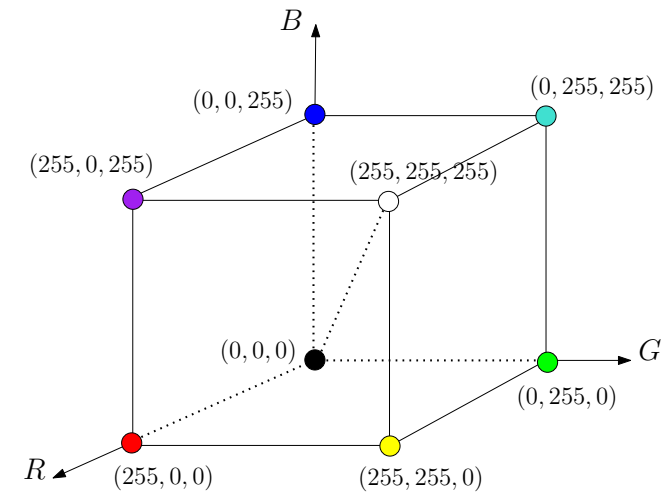

Figure 4: The range of colors represented on the Cartesian 3-dimensional system.

For example, let $(240,29,35)$ be the color of the upper left pixel of an image $I$, i.e., $I(1,1)=$ $(240,29,35)$. Then, in our system $\operatorname{Img}(1,1,1)=240$, $\operatorname{Img}(1,1,2)=29$, and $\operatorname{Img}(1,1,3)=35$.

\section{OUR WATERMARKING SYSTEM}

In this section we describe the modules and the main functions of the proposed watermarking system. Our system, which we named WaterIP, provides to a student two main working levels:

- Embed level: Through a friendly graphical user interface, the student creates a secret key (i.e., the watermark $w$ ) and selects a picture $I$ in which he wants to embed the watermark; in our system the watermark $w$ is a permutation $\pi$ over the set $N_{6}$ and it is embedded into the original picture $I$ (see, Figure 6), using the 2DM representation, resulting the watermarked picture $I_{w}$ (see, Figure 7).

- Mark level: The student, in order to prove that he is the owner of the picture $I_{w}$, inputs the watermarked picture $I_{w}$ into the system which makes the marks visible to the student so that he will be able to easily extract the watermark $w$ (i.e., his secret key) just by looking at the marks; in particular, the system returns the marked picture $I_{m}$ to the student (see, Figure 8).

Our WaterIP system consists of two main components. The usability of the system is based on a friendly to the student graphical user interface. Using it he can easily produce his watermark $w$, i.e., a permutation $\pi$, using his mouse without making any mistake. He can also choose an image $I$ from his computer and he can either embed a watermark into $I$ resulting the watermarked image $I_{w}$ or make the marks 
of $I_{w}$ visible so that he will be able to prove to his teacher that the picture belongs to him.

The first component is responsible for embedding the desired watermark $w=\pi$ into the image $I$ using the 2DM representation of $\pi$, while the second one is responsible for making the marks of a watermarked image $I_{w}$ visible to the student so that he will be able to easily extract the watermark $w$ by hand.

There could have been a third component for extracting the permutation from the watermarked image but we chose not to include it because we consider important, for pedagogical reasons, that the student must participate interactively in the process of proving ownership.

We should mention that our system uses a permutation $\pi$ over the set $N_{6}$ for the watermark $w$. The set $N_{6}$ was selected in purpose; we preferred to use a fixed size rather than giving the size as a choice to the user. That choice was made in order to make the system simple as it is designed for educational reasons. Also 6 is not a great number so it is relatively easy to memorize by a student, nor it is a very small number and that makes the permutation more presentable.

The system is organized into subsystems, each of which contains an algorithm responsible for a particular operation of WaterIP (Sommerville, 2010; Gamma et al., 1995; Taylor et al., 2009). The two subsystems of our system that are considered the basic ones, are those responsible for embedding the watermark and marking the image.

All the system's algorithms have been developed and tested in MATLAB, ver. R2008b. Also the decompression and compression of the images were done using the imread and imwrite functions of MATLAB's library (Gonzalez et al., 2003).

\subsection{Embed Watermark into Image}

We next describe an algorithm which embeds a permutation $\pi$ into an image $I$; recall that, in our system we use a permutation $\pi$ over the set $N_{6}$ for the watermark $w$ (Sedgewick and Flajolet, 1996; Golumbic, 1980); see, also Subsection 2.1.

The algorithm takes as input a permutation $\pi$ and an image $I$, in which the user wants to embed the watermark $w=\pi$, and produces the watermarked image $I_{w}$; it works as follows:

Step 1: The algorithm first computes the 2DM representation of the permutation $\pi=\left(\pi_{1}, \pi_{2}, \ldots, \pi_{6}\right)$, that is, it computes the $6 \times 6$ array $A$ (see, Subsection 2.1); the entry $\left(i, \pi_{i}\right)$ of the array $A$ contains the symbol "*", $1 \leq i \leq 6$.

Step 2: Next, the algorithm takes the input image $I$ and places on it an imaginary $6 \times 6$ grid which covers the whole images. Then it scans the image and goes to each grid-cell $C_{i j}(I)$ (there are always 36 gridcells in any image) and locates the central pixel $p_{i j}^{0}$ of the grid-cell $C_{i j}(I), 1 \leq i, j \leq 6$.

Then, it computes the difference between the brightness of the central pixel $p_{i j}^{0}$ and the average brightness of the eight pixels $p_{i j}^{1}, p_{i j}^{2}, \ldots, p_{i j}^{8}$ around it, and stores this value in the variable $\operatorname{dif}\left(p_{i j}^{0}\right)$ (see, Figure 5).

Finally, it computes the maximum absolute value of all $36 \operatorname{differences} \operatorname{dif}\left(p_{i j}^{0}\right), 1 \leq i, j \leq 6$, and stores it in the variable $\operatorname{Maxdif}(I)$.

Step 3: The algorithm goes again to each central pixel $p_{i j}^{0}$ of each grid-cell $C_{i j}$ and if the corresponding entry $A(i, j)$ contains the symbol “*”, then it increases its brightness $k_{i j}^{0}$ by the value $e_{i j}^{0}$ so that it surpasses the image's maximum difference $\operatorname{Maxdif}(I)$ by a constant $c$; that is, $k_{i j}^{0}+e_{i j}^{0}=\operatorname{Maxdif}(I)+c$.

In our system we use $c=9$, and thus the brightness $k_{i j}^{0}$ of the central pixel of each grid-cell $C_{i j}$ is increased by $e_{i j}^{0}$, where

$$
e_{i j}^{0}=\operatorname{Maxdif}(I)-k_{i j}^{0}+9
$$

$1 \leq i, j \leq 6$.

Let $I_{w}$ be the resulting image after increasing the brightness of the 6 central pixels, with respect to $\pi$, of the image $I$. Hereafter, we call these 6 central pixels as 2DM-pixels; recall that, $p_{i j}^{0}$ is a 2DM-pixel if $A\left(i, \pi_{i}\right)=$ "*”, or, equivalently, the cell $\left(i, \pi_{i}\right)$ of the matrix $A$ is marked.

Step 4: The algorithm returns the watermarked image $I_{w}$.

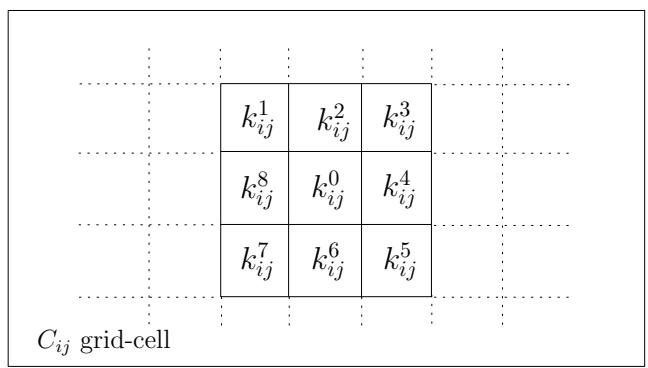

Figure 5: The brightness $k_{i j}^{\ell}$ of the pixels $p_{i j}^{\ell}, \ell=0,1, \ldots, 8$.

Having described our system's algorithm which embeds a permutation $\pi$ into an image $I$, let us now show the efficiency of our algorithm by computing its time and space complexity.

Complexity. We shall compute the complexity of each step of the algorithm; suppose that the input image $I$ has $N \times M$ size (i.e., pixels). 
It is easy to see that the Step 1 requirers (asymptotic) constant time and space, since the length of the permutation $\pi$ and the size of the array $A$ are 6 and $6 \times 6$, respectively.

In Step 2 the algorithm places on $I$ an imaginary $6 \times 6$ grid. To this end, the values of the two dimensions of the image $I$ must be known, and thus this computations takes $N+M$ time; note that, the algorithm takes the image $I$ as an $N \times M$ array. Then, the location of the 36 central pixels $p_{i j}^{0}$ can be done in constant time, $1 \leq i, j \leq 6$.

The difference between the brightness of the central pixel $p_{i j}^{0}$ and the average brightness of the eight pixels around it, denoted by $\operatorname{dif}\left(p_{i j}^{0}\right)$, is computed as follows:

where,

$$
\operatorname{dif}\left(p_{i j}^{0}\right)=\left|k_{i j}^{0}-\frac{\sum_{\ell=1}^{8} k_{i j}^{\ell}}{8}\right|
$$

$$
k_{i j}^{\ell}=\frac{x_{i j}^{\ell}+y_{i j}^{\ell}+z_{i j}^{\ell}}{3}
$$

Recall that, the values $x_{i j}^{\ell}, y_{i j}^{\ell}$, and $z_{i j}^{\ell}$ compose the brightness $k_{i j}^{\ell}$ of the pixel $p_{i j}^{\ell}$ in the $R G B$ model (see, Subsection 2.2). Thus, the $36 \operatorname{differences} \operatorname{dif}\left(p_{i j}^{0}\right)$ can be computed in constant time and require constant space (i.e., an array of $6 \times 6$ size).

Finally, in this step the algorithm computes the maximum absolute value $\operatorname{Maxdif}(I)$ of all 36 differences $\operatorname{dif}\left(p_{i j}^{0}\right)$, that is,

$$
\operatorname{Maxdif}(I)=\max \left\{\operatorname{dif}\left(p_{i j}^{0}\right) \mid 1 \leq i, j \leq 6\right\}
$$

which obviously takes also constant time.

The only operation performed in Step 3 is the increment of the brightness $k_{i j}^{0}$ of each central pixel by the value $e_{i j}^{0}$ (see, Equation 1 ); it obviously takes constant time since there are 36 such central pixels.

Thus, based on the step-by step complexity analysis we conclude that our embedding algorithm runs in $O(N+M)$ time and requires $O(1)$ space, where $N, M$ are the two dimensions of the input image $I$.

Remark 1. The values $x_{i j}^{\ell}, y_{i j}^{\ell}$, and $z_{i j}^{\ell}$ which compose the brightness $k_{i j}^{\ell}$ of the pixel $p_{i j}^{\ell}$ are stored in the array Img at the entries $\left(i^{\prime}, j^{\prime}, 1\right),\left(i^{\prime}, j^{\prime}, 2\right)$, and $\left(i^{\prime}, j^{\prime}, 3\right)$, respectively (see, Subsection 2.2). Note that, $\left(i^{\prime}, j^{\prime}\right)$ is the position of pixel $p_{i j}^{\ell}$ in image $I$, while $(i, j)$ is the position of pixel $p_{i j}^{\ell}$ in the $6 \times 6$ grid.

\subsection{Show Marks on Image}

Next we describe our system's algorithm which is responsible for making the marks of a watermarked im-

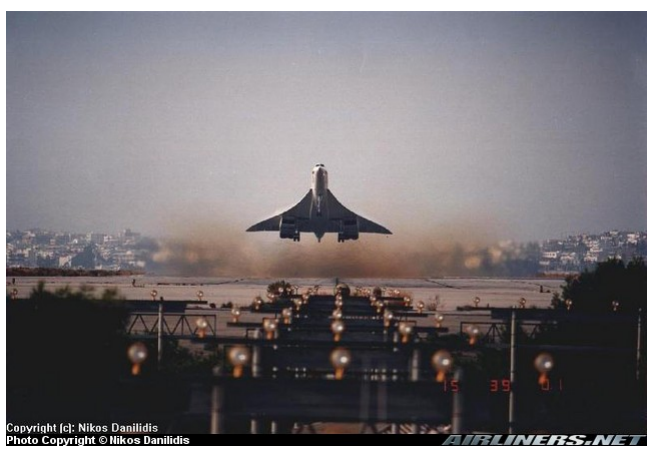

Figure 6: The original image $I$.

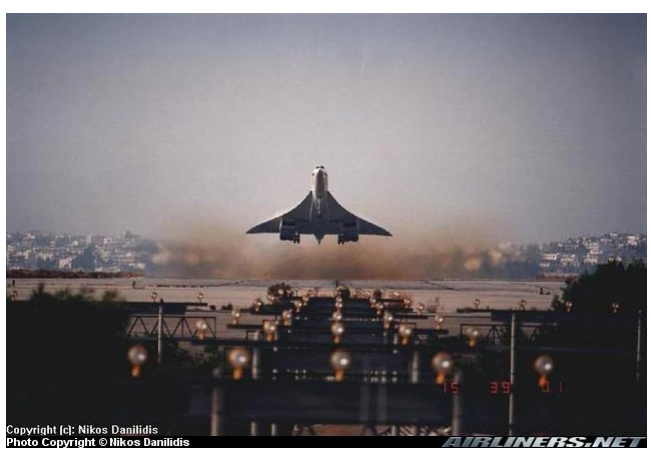

Figure 7: The watermarked image $I_{w}$.

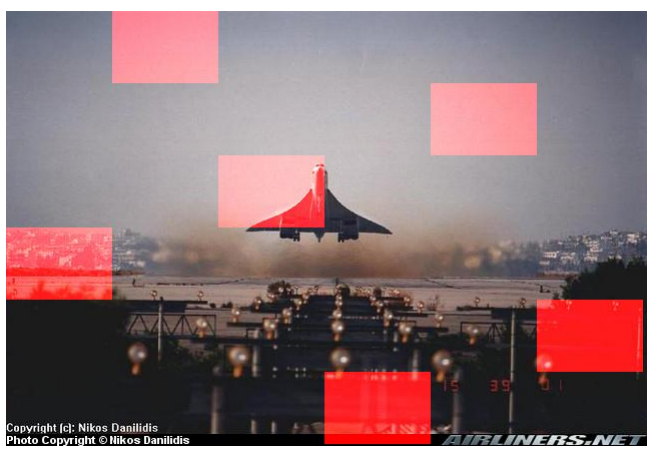

Figure 8: The marked image $I_{m}$.

age $I_{w}$ visible to the student so that he will be able to extract, in a specific way, the watermark $w$ by hand.

The algorithm takes as input a watermarked image $I_{w}$ in which the student wants to make the markings visible, and produces the marked image $I_{m}$; the steps of the algorithm are the following:

Step 1: The algorithm places again the same imaginary $6 \times 6$ grid on image $I_{w}$ and locates the central pixel (the 2DM-pixel) $p_{i j}^{0}$ of each grid-cell $C_{i j}(I)$, $1 \leq i, j \leq 6$; there are 36 central pixels in total. Then, it finds the 6 central pixels $p_{1}^{0}, p_{2}^{0}, \ldots, p_{6}^{0}$, among the 
36 , with the maximum brightness using a known sorting algorithm.

Step 2: In this step, the algorithm takes the 6 gridcell $C_{1}, C_{2}, \ldots, C_{6}$ of the image $I_{w}$ which correspond to 6 central pixels $p_{1}^{0}, p_{2}^{0}, \ldots, p_{6}^{0}$, and places a red film over the whole area of each grid-cell $C_{i}, 1 \leq i \leq 6$.

To this end, the algorithm takes each pixel $p_{i, j}$ of each grid-cell $C_{i}, 1 \leq i \leq 6$, and modifies the brightness $k_{i, j}=\left(x_{i, j}, y_{i, j}, z_{i, j}\right)$ of the pixel $p_{i, j}$ as follows:

$$
k_{i, j}^{\prime}=\left(255, y_{i, j}, z_{i, j}\right)
$$

where, $j$ takes $N / 6 \times M / 6$ different values. The resulting image is the marked image $I_{m}$ (see, Figure 8).

Step 3: Finally, the algorithm returns the marked image $I_{m}$.

Let us next compute the time and space efficiency of the proposed algorithm by computing the complexity of each step separately.

Complexity. Again, suppose that the input image $I_{w}$ has $N \times M$ size (i.e., pixels).

In Step 1 the algorithm places on $I_{w}$ an imaginary $6 \times 6$ grid, as the embedding algorithm do on image $I$, and thus the values of the two dimensions of the image $I_{w}$ must be known; this computations takes $N+M$ time. Then, the location of the 36 central pixels $p_{i j}^{0}$ can be done in constant time, $1 \leq i, j \leq 6$, while the finding of the 6 central pixels, among the 36, with the maximum brightness can also be done in constant time; note that, it is well known that any sorting algorithm on input of constant size is executed in constant time.

In Step 2 the algorithm takes the 6 grid-cell $C_{1}, C_{2}, \ldots, C_{6}$ of the image $I_{w}$ which correspond to the 6 central pixels with maximum brightness, and modifies the brightness of all their pixels. The 6 grid-cell contain $\frac{N \times M}{6}$ pixels in total. Thus, Step 2 requires $O(n)$ time, where $n=N \times M$.

Based on the above complexity analysis we conclude that the proposed marking algorithm runs in $O(n)$ time and requires $O(1)$ space, where $n$ is the number of pixels of the input image $I_{w}$.

\subsection{Performance}

The main two components of our system are (i) the algorithm for embedding a watermark $w$ into an image $I$ resulting the image $I_{w}$, and (ii) the algorithm for making the watermark marks of $I_{w}$ visible resulting the image $I_{m}$.

We next discuss some issues concerning the performance of the system; in particular, we mainly focus on the efficiency of watermarking image $I_{w}$ produced by the embedding algorithm and also on the time and space complexity of the two main algorithms (i.e., the embedding and the marking algorithms).

We have evaluate our embedding algorithm by testing it on more that 100 images selected from various websites and we are in a position to claim that the watermarking technique used by the algorithm can be considered efficient because the watermark $w$ is hidden very well in the images $I_{w}$; in other words, after an image has been watermarked we can not figure out by looking at it where exactly it has been marked.

We believe that the watermark $w$ is well hidden in image $I_{w}$ because we mark the image by changing the difference between the brightness of the central pixel of each cell of the $6 \times 6$ imaginary grid and its 8 neighborhood pixels (see, Step 2 of the embedding algorithm). Among the 36 central pixels of the image we consider as marked the 6 cells that have the maximum difference. Note that, when we change this difference to mark the cell, we make it equal to the maximum difference of all the 36 central pixels plus the value 9 . We add nine because if we compress the image the values of the pixels may slightly change, and we want our watermark to be robust. We also believe that this technique despite being simple, it is efficient because the brightness of each of the 6 marked central pixels does not have a great difference anyway from the brightness of the 8 neighborhood pixels and thus the modified central pixel does not change something significantly in the image.

As far as the time and space complexity of our system is concerned, we should mention that it is asymptotically linear in the size (i.e., number of pixels) of the input images.

More precisely, the embedding algorithm is very fast, it has almost constant time complexity since it operates only on the 36 grid-cells of the input image $I$. Note that, in our implementation the length of the watermark is 6 and thus we always have 36 grid-cells. Expressing the algorithm's complexity by the size of the input image $I$, we can say that it is of order $N+M$, where $N, M$ are the two dimensions of $I$.

The marking algorithm is also very fast since it also operates mainly on the 36 grid-cells of the input image $I_{w}$. The most time consuming step of the algorithm is that of placing a red film over the whole area of each of the 6 marked grid-cell $C_{i}, 1 \leq i \leq 6$. This step takes $O(n)$ time, where $n$ is the number of the pixels of $I_{w}$ (in fact, it requires $(N \times M) / 6$ operations); recall that, $n=N \times M$.

Finally, it is fair for the time performance of our system to take into consideration the time needed for decompressing the image $I$ that the system takes as input; note that, the system usually works with compressed images as input. It is obvious that the time 
needed for decompressing the image $I$ into a raw raster format depends on the type of the image selected. The most common types of images would be the JPEG as digital cameras store images of this type and also nearly every image on the World Wide Web is in JPEG format. The compression or decompression of a JPEG requires the usage of the DCT (discrete cosine transform) or the iDCT (inverse discrete cosine transform), respectively. The DCT is similar to a Fourier transform and it is of order $n^{2}$, but it is also possible to do the same thing by doing something similar to the FFT (fast fourier transform) which is of order $n \log n$. Note that the same techniques applies for the JIF images which are also popular in the web (Ahmed et al., 1974; Cooley and Tukey, 1965).

Summarizing, the total asymptotic time performance of our system is $O(n \log n)$ for decompressing the input image $I, O(N+M)$ for embedding the watermark $w$ into $I$, and $O(n)$ for marking the image $I_{w}$ and producing the image $I_{m}$, where $n$ is the number of the pixels of the input image and $n=N \times M$. Moreover, the extra space needed by the system is constant, i.e., it uses only some extra auxiliary variables.

\section{INSIDE THE CLASSROOM}

Our work proposes a supporting educational tool for teaching, inside the classroom, the value of intellectual property. As mentioned before we believe that the best way to gain people's respect towards intellectual property rights is to start from the roots, and that is the early education. Students should be taught, as part of the ethical education, to respect intellectual property at schools (Watkins et al., 2007).

We believe that a student can easier understand what is the notion behind intellectual property, if himself experiences an example of his own intellectual property being theft and then having to find a way to claim it. Teaching intellectual property only in theory is not pedagogically enough and that's because it's a too technical term for a student to understand, whereas an interactive method, which combines theory and real experience will be without any doubt much more effective. That can be illustrated by using our WaterIP educational watermarking tool (Smaldino et al., 2007; Kyriacou, 2009).

We consider that our tool can be applied in all educational levels, as part of various lesson plans, beginning from early childhood; uploading an image or photo the teacher can demonstrate the system to preschool learners and set them ethical dilemmas concerning the proper use of images or photos. Teachers of preliminary and high schools can go on more com- plex meanings and explain learners the concepts of watermarking, embedding, extracting, permutation, image analysis, etc.

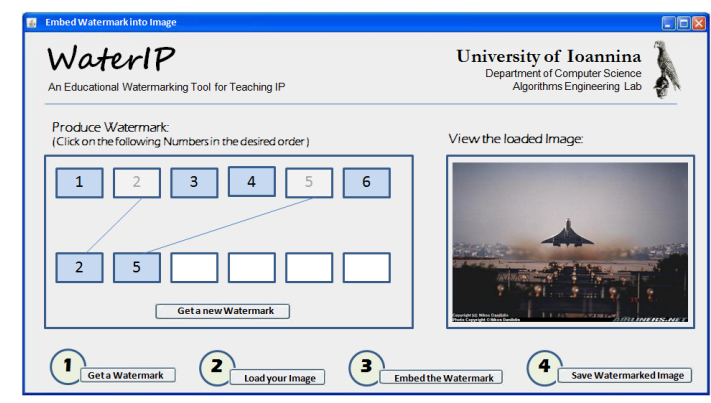

Figure 9: Part of the WaterIP interface.

We next use an imaginary scenario where two classmates claim the ownership of the same image, Alex who is really the owner of the image and Bob who claims that he is the owner, and show how our WaterIP system helps both to understand what intellectual property rights really stand for. Then we recommend a lesson plan of how our WaterIP system can be efficiently applied in groups inside a classroom during a course.

\subsection{Two Classmates}

Let us first present the scenario of the two classmates, Alex and Bob, where both claim the ownership of the image of Figure 7.

The main idea of the scenario is, that Alex first paints a picture at a computer or just takes a photo and uploads it to the computer. Afterwards he runs our system and places the numbers between 1 and 6 in a random order that he memorizes and keeps secret. That order actually forms the permutation which also is the watermark that Alex wants to embed in his digital image. He selects from a menu which image he wants to use and then runs the algorithm and places the watermark into it. Then he gets the watermarked image. That watermark will later be his proof that the picture was really made by him and that he deserves to be rewarded for it and not someone else; of course we shall not forget that in order to do that he should have memorized the permutation in order to claim the property of the image. Afterwards Alex uploads the watermarked image in his personal student web-page or in the lesson's webpage, making it public to every student.

In Figure 9 we show a form, part of the system's friendly user interface, in which Alex produces the watermark that he wants to embed in his image. What he does here is that he selects the numbers from the 
upper set by clicking on them and places them linearly in the second set of numbers which forms the watermark (Button 1) and then selects and views an image (Button 2); the figure depicts an intermediate stage where Alex has already load an image and has selected the fist two numbers, i.e., 2 and 5, of his watermark. After the creation of his watermark, he is able to embed it in the image (Button 3) and save the watermarked image (Button 4).

Then Bob may later download the picture and insist to the teacher that he painted it. Now the teacher should pretend that he is going to reward that student for that picture but also asks him if he can prove that it is really his image. Of course Alex that really painted that image would not want another one to be awarded with a grade for a picture that he painted. So Alex also gets into the discussion by telling his teacher that there is a watermark in that picture and also which is the value of this watermark. This way he can prove that it is his property whereas Bob won't be able to do that. Then Alex uses the WaterIP program again, inserts the picture that is being claimed by both sides and gets the marked picture. Last Alex applies the extracting algorithm, gets printed the marked image, and using a ruler for his help gets the permutation. That proves to the teacher that the picture was indeed painted by Alex as the watermark he was told previously matches with the one that was just extracted from the picture. Last the teacher rewards Alex for his picture.

The above process is demonstrated at Figures 10, 11 , and 12. Figure 10 demonstrates an imaginary discussion between the two classmates: Bob who insists that he is the owner of a picture and Alex who claims that he is the real owner and that Bob isn't. Of course Alex also says that he has a proof that the picture is his property and that proof lies in the watermark that he embedded previously in the picture. He also shows that watermark to Bob. Figure 11 shows the most important part of the whole process. After Alex runs the marking algorithm he has a picture with red films over the marked areas and, using a ruler and a pencil, he follows the extracting procedure:

\section{Procedure Water_Extract}

1. Scan the picture with the ruler from left to right and write the numbers from 1 to 6 in ascending order inside the red films;

2. Get the watermark by scanning the picture from top to bottom and read every number that comes across;

Figure 12 shows Alex pointing out that the watermark he initially had matches the one extracted from

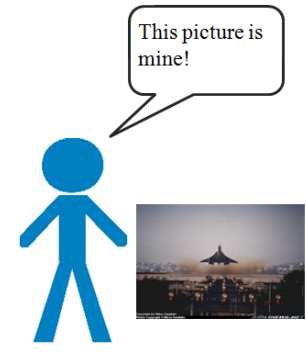

Bob

Figure 10: Bob claims that he is the owner of the picture.

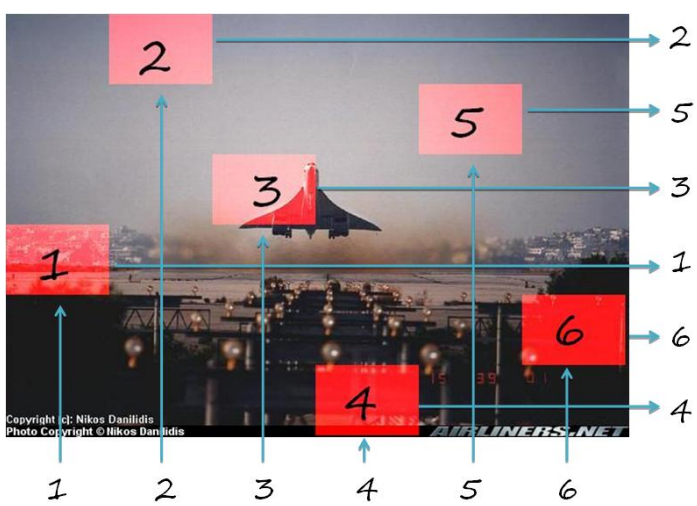

Figure 11: The marked image $I_{m}$.

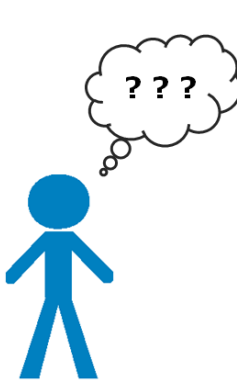

Bob

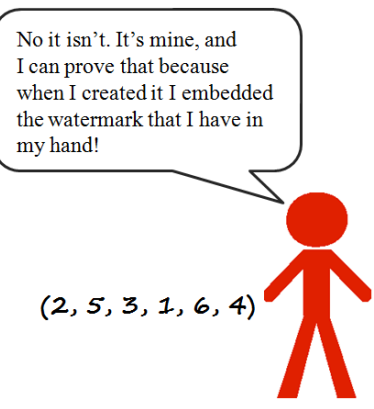

Alex
Figure 12: Alex proves that he is the owner of the picture.

the picture meaning he is the owner and last he advises Bob to respect intellectual property from now on. That makes Bob think again for what he just did.

It is worth mentioning that after the completion of the above process a student has experienced, he can then easier understand what intellectual property really stands for. The teacher should tell the students after that experience that this is why intellectual property should be protected and as he now has the interest of the students he may now teach them about intel- 
lectual property and the problems around them as the students now know that this issue concerns them as well.

\subsection{A Course Design}

We next recommend a lesson plan of how our WaterIP system may be applied during a course. We suppose that we have a class of 15 students and the teacher is to incorporate intellectual property content into his lecture to enable students to consider how to respect others' intellectual property rights, how to protect their own ideas and how they can legitimately make use of others' intellectual property; we briefly describe an overview of a lesson plan.

At the beginning, the teacher presents the learning objectives of the course concerning intellectual property and then he explains to his students that intellectual property can be protected through technical means (Anderson et al., 2001; Marzano and Kendall, 2007; Simon and Taylor, 2009; Harden, 2002); note that, sometimes learning objectives referred to as learning outcomes or course-specific goals.

After that the teacher introduces the WateIP system for watermarking images and demonstrates the main operational parts of it. At this stage, based on students' developmental characteristics, he can analyze the most important technical terms, such as, watermark, secret key, pixels, image analysis, eye perception, embed, extract, prove ownership, etc.

The teacher uses a role-playing method to involve students to teaching process through action; we briefly say that, the role-play process provides students with an opportunity to explore their feelings, gain insight about their attitudes, and increase problem solving skills (Joyce et al., 2000; Emmer et al., 2002; Evertson et al., 2002).

In our scenario, the teacher and his students work inside the classroom as follows:

Action A: The teacher divides his classroom into student groups of five persons each and assigns roles to groups as follows:

- the first is the true ownership claimer group,

- the second is the false ownership claimer group,

- and the third one is the law group.

The students in the true group (or, active group) will be the creators of the images, the students in the false group will claim the ownership of the images created by the true group, while the law group will handle the conflict between the true and false owners.

Action B: The teacher propose an imaginary scenario where true and false owners are photographers and gives to each one of the two groups the task to take, draw or scan images using cameras and/or computers, to watermark them by using the WaterIP tool, and then to upload the watermarked images to a photo gallery, i.e., a specially designed website public for everyone, for collecting the images. But also tells them that the false group should not upload any image and claim later that one of the already uploaded in the photo gallery belongs to them.

Action C: The teacher opens the photo gallery and asks for each image which is the group that uploaded it in order to award it with a symbolic grade. Of course we end up with one image claimed by two groups. Now what the law group does is asking first the students of the two groups to tell which watermark they embedded into the image. Then, a student of the law group runs WaterIP and makes the image's marks visible. Next he prints the marked image into a paper and the law group extracts the watermark using a ruler and a pencil according to Procedure Water_Extract; see, Subsection 4.1. The teacher and his students then realize which group tells the truth and the teacher awards it with a grade.

After the actions A, B, and C fulfil the teacher may discuss with the groups what they learnt from that experience. That might be done from the point of view of the true group, the false group, and the law group that witnessed the conflict. The teacher based on the experience that the students just had explains them more things about intellectual property. Moreover, the teacher can also put into consideration ethical issues concerning the economic and psychological effects of stealing someone else's creation and discuss it with all groups. Last the teacher can write all the conclusions of this discussions on the board.

At the end, the teacher asks the true group that managed to prove the ownership, how they would have felt if they had not that privilege to do that.

\section{CONCLUDING REMARKS}

In this paper, we proposed a new pedagogical tool that provides a step by step demonstration of embedding and extracting watermarks into images defined by the user in the form of permutations. Our system, named WaterIP, incorporates concepts from graph theory (2D representations of permutations) and image processing (editing values of space domain), it has a friendly graphical user interface, and it is designed to be used to support the teaching and learning process on intellectual property rights. We also demonstrated its educational effectiveness by presenting ways of how it can be applied in class. 
It is worth noting that although our system's target group, as described in this paper, is children aged between 9 and 12 years, the watermarking method behind WaterIP can be applied to all educational levels. Indeed, it would not be difficult to design alterative modules, regarding mainly the type of the watermark that a student creates, that can be implemented in order to make it accessible by different ages as well. For example a version using objects instead of numbers for representing the elements of the permutation, can be developed in order to be used by younger than 9 years old children. Furthermore a version using integers as an input and algorithms for converting integers into permutations, could be designed in order to support the teaching process on intellectual property, through image watermarking, to older ages as well; we leave such a system's extension as a topic for further investigation.

Furthermore, in light of our two scenarios presenting ways of how our WaterIP system can be applied inside a class it would be very interesting to come up with new scenarios in order to gain more information about its educational effectiveness. A typical idea for such a scenario is that we could divide children into 2 groups and then teach the first group intellectual property issues using our system as a supportive tool, and then teach the second group the same content only theoretically; last evaluate each group's knowledge gained and compare the results.

\section{REFERENCES}

Ahmed, N., Natarajan, T., and Rao, K. R. (1974). Discrete cosine transform. IEEE Transactions on Computers, C-23:90 - 93 .

Anderson, L. W., Krathwohl, D. R., Airasian, P. W., Cruikshank, K. A., Mayer, R. E., Pintrich, P. R., Raths, J., and Wittrock, M. C. (2001). A Taxonomy for Learning, Teaching and Assessing: A Revision of Blooms Taxonomy of Educational Objectives. Longman, New York.

Collberg, C. and Nagra, J. (2010). Surreptitious Software. Addison-Wesley.

Cooley, J. W. and Tukey, J. W. (1965). An algorithm for the machine calculation of complex fourier series. Mathematics of Computation, C-23:297 - 301.

Cox, I., Kilian, J., Leighton, T., and Shamoon, T. (1996). A secure, robust watermark for multimedia. In Proc. 1st Int'l Workshop on Information Hiding, volume LNCS 1174, pages $317-333$.

Davis, J. C. (1997). Intellectual property in cyberspace what technological / legislative tools are necessary for building a sturdy global information infrastructure? In IEEE Int'l Symposium on Technology and Society, pages $66-74$.
Emmer, E., Evertson, C., Clements, B., and Worsham, W. (2002). Classroom Management for Secondary Teachers. Prentice-Hall, 6th edition.

Evertson, C., Emmer, E., Clements, B., and Worsham, W. (2002). Classroom Management for Elementary Teachers. Prentice-Hall, 6th edition.

Gamma, E., Helm, R., Johnson, R., and Vlissides, J. (1995). Design Patterns: Elements of Reusable Object-Oriented Software. Addison-Wesley.

Golumbic, M. (1980). Algorithmic Graph Theory and Perfect Graphs. Academic Press, Inc., New York.

Gonzalez, R. C. and Woods, R. E. (2007). Digital Image Processing. Prentice-Hall, 3rd edition.

Gonzalez, R. C., Woods, R. E., and Eddins, S. L. (2003). Digital Image Processing Using Matlab. PrenticeHall.

Grover, D. (1997). The Protection of Computer Software Its Technology and Applications. Cambridge University Press, New York.

Harden, R. M. (2002). Learning outcomes and instructional objectives: is there a difference? Medical Teacher, 24:151 - 155 .

Jain, K., Raghavan, M., and Jha, S. K. (2009). Study of the linkages between innovation and intellectual property. In Proc. of PICMET 2009, pages 1945 - 1953.

Joyce, B., Weil, M., and Calhoun, E. (2000). Models of Teaching. Prentice-Hall, 6th edition.

Kyriacou, C. (2009). Teaching in Schools: Theory and Practice. Nelson Thornes, 3nd edition.

Lemley, M. A. (2005). Intellectual property, and free riding. Texas Law Review, 83: 1031:1033.

Marzano, R. J. and Kendall, J. S. (2007). The New Taxonomy of Educational Objectives. Corwin Press, CA.

Pascale, D. (2003). A Review of RGB Color Spaces ...from $x y Y$ to $R^{\prime} G$ ' $B$ '. The BabelColor Company.

Raysman, R., Pisacreta, E. A., and Adler, K. A. (1999). Intellectual Property Licensing: Forms and Analysis. Law Journal Press.

Sedgewick, R. and Flajolet, P. (1996). An Introduction to the Analysis of Algorithms. Addison-Wesley.

Simon, B. and Taylor, J. (2009). What is the value of coursespecific learning goals? Journal of College Science Teaching, Nov/Dec:53 - 57.

Smaldino, S. E., Lowther, D. L., and Russell, J. D. (2007). Instructional technology and media for learning. Prentice-Hall, 9nd edition.

Sommerville, I. (2010). Software Engineering. AddisonWesley, 9th edition.

Tamada, H., Nakamura, M., and Monden, A. (2004). Design and evaluation of birthmarks for detecting theft of java programs. In Proc. Int'l Conference on Software Engineering (SE'04), pages $569-575$.

Taylor, R. N., Medvidovic, N., and Dashofy, E. M. (2009). Software Architecture: Foundations, Theory, and Practice. Wiley.

Watkins, C., Carnell, E., and Lodge, C. (2007). Effective Learning in Classrooms. Sage Publications, 1nd edition. 